\title{
A Programação Pactuada e Integrada como instrumento de garantia da integralidade da atenção à saúde no SUS
}

\author{
The Agreed and Integrated Programming as an instrument to guarantee the \\ integrality of health care in the SUS
}

\section{La Programmation Concertée Integrée comme instrument de garantie l'intégralité soins de santé en le SUS}

\section{La Programación Acordada Integrada como instrumento de garantía de la integración de la atención a la salud en el SUS}

\author{
Lenice Carrilho de Oliveira Moreira ${ }^{1}$ \\ Edson Mamoru Tamaki ${ }^{1}$ \\ Recebido em 09/12/2016; revisado e aprovado em 26/04/2017; aceito em 05/05/2017 \\ DOI: http://dx.doi.org/10.20435/inter.v18i4.1454
}

\begin{abstract}
Resumo: O estudo analisou a Programação Pactuada e Integrada (PPI) como instrumento de garantia de acesso da população às ações e serviços de saúde em oito municípios sul-mato-grossenses, utilizando uma abordagem qualitativa exploratória. Os dados foram coletados por meio de entrevistas semiestruturadas e análise documental. Os resultados permitiram concluir que a PPI não está desempenhando o seu papel de instrumento garantidor do acesso da população a serviços de maior complexidade.
\end{abstract}

Palavras-chave: gestão em saúde; planejamento em saúde; Programação Pactuada e Integrada; regionalização. Abstract: The study analyzed the Agreed and Integrated Programming (PPI) as an instrument to guarantee the population's access to health actions and services in eight counties of South-Mato Grosso, using a qualitative exploratory approach. Data were collected through semi-structured interviews and documentary analysis. The results allowed to conclude that PPI is not performing its role as an instrument to guarantee the access of the population to services of greater complexity.

Key words: management health; health planning; Agreed and Integrated Programming; regionalization.

Résumé : L'étude a analysé la Programmation Concertée Integrée (PPI) comme instrument de garantie d'accès aux actions et services de santé dans huit municipalités de Mato Grosso du Sud, en utilisant une approche qualitative exploratoire. Les données ont été recueillies au moyen d'entrevues semi-structurées et d'analyse de documents. Les résultats ont montré que la PPI ne remplit pas son rôle d'instrument de garantie d'accès de la population à des services plus complexes.

Mots-clés: gestion de la santé; planification de la santé; Programmation Concertée Integrée; regionalisation. Resumen: El estudio analizó la Programación Acordada Integrada (PAI) como instrumento de garantía de acceso de la población a las acciones y servicios de salud en ocho municipios de Mato Grosso del Sur, utilizando un enfoque exploratorio cualitativo. Los datos fueran recolectados por medio de entrevistas semiestructuradas y análisis de los documentos. Los resultados permitirán concluir que la PAI no está desempeñando su papel de instrumento garantizador del acceso de la población a servicios de más complejos.

Palabras clave: gestión de la salud; planificación en salud; Programación Acordada Integrada; regionalización.

\section{INTRODUÇÃO}

O Sistema Único de Saúde (SUS), criado pela Constituição Federal de 1988 e regulamentado pelas Leis n. 8.080/90 e n. 8.142/90, constituiu-se no modelo oficial público de atenção à saúde no Brasil. A política de saúde definida constitucionalmente foi orientada pelos princípios da universalidade e da igualdade no acesso a ações e serviços, os quais deveriam integrar uma

\footnotetext{
${ }^{1}$ Universidade Federal de Mato Grosso do Sul, Campo Grande, Mato Grosso do Sul, Brasil.
} 
rede regionalizada e hierarquizada, constituindo um sistema único (BRASIL, 1988), a partir da formação de redes assistenciais (SILVA, 2011).

O SUS pressupõe a distribuição de responsabilidades, encargos e atribuições entre os entes federados, enfatizando descentralização dos serviços para os municípios e a cooperação e articulação entre os níveis de governo (AMORIM; MENICUCCI, 2008). Dessa forma, um novo desafio foi imposto aos gestores do SUS, que deveriam adotar mecanismos eficazes de regionalização e hierarquização da atenção à saúde, com vistas a efetivar o princípio da integralidade (VENÂNCIO et al., 2011).

Com a ampliação da responsabilização do Estado em relação às ações e serviços de saúde, fez-se necessária a adoção de estratégias capazes de promover máxima eficiência ao processo de gestão, assumindo a atividade de planejamento, papel fundamental na racionalização da organização, acompanhamento e avaliação da atuação sistêmica do SUS (BRASIL, 2008).

De acordo com a legislação, o processo de planejamento e orçamento do SUS será ascendente, partindo do âmbito local até o federal e, ouvidos os respectivos Conselhos de Saúde, deverá compatibilizar as necessidades das políticas de saúde com a disponibilidade de recursos em planos de saúde (BRASIL, 1990; 2011c).

A regionalização é a diretriz do SUS que orienta a descentralização das ações e serviços de saúde, bem como os processos de negociação e pactuação entre os gestores (BRASIL, 2006a). A articulação entre os gestores municipais para pactuação das referências intermunicipais dar-se-á por meio da Programação Pactuada e Integrada (PPI) (BRASIL, 2011b).

A PPI é o instrumento que, em consonância com o processo de planejamento, visa definir e quantificar as ações de saúde para a população residente em cada território, além de nortear a alocação dos recursos financeiros a partir de critérios e parâmetros pactuados entre os gestores. Deve, ainda, explicitar os pactos de referência entre municípios e definir a parcela de recursos destinados à assistência da própria população e da população referenciada por outros municípios (BRASIL, 2006b).

De acordo com Molesini et al. (2010), a elaboração da programação deve observar critérios e parâmetros estabelecidos pelas Comissões Intergestores e ser aprovada pelos respectivos conselhos, devendo, sua finalização, expressar a distribuição de recursos, inclusive os repassados pelo Ministério da Saúde.

Para Venâncio et al. (2011, p. 3952), a PPI

[...] é a conjugação dos recursos técnicos de saúde existentes, voltando-os ao atendimento integrado das necessidades, cuja pactuação se dá entre entes políticos autônomos, pressionados por interesses diversos, os quais muito frequentemente não representam as necessidades da população.

Assim sendo, o processo de PPI, coordenado pelo gestor estadual, representa o principal instrumento para a garantia de acesso da população aos serviços de média complexidade não disponíveis em seu município de residência, além de orientar a alocação de recursos e a definição de limites financeiros para todos os municípios do estado, independente de sua condição de gestão (BRASIL, 2001).

Em 2006, as diretrizes operacionais do Pacto de Gestão, uma das dimensões do Pacto pela Saúde, reafirmaram princípios e consolidaram processos, como a importância da regionalização e dos instrumentos de planejamento, destacando-se, dentre esses, o Plano Diretor de Regionalização (PDR), o Plano Diretor de Investimentos (PDI) e a Programação Pactuada e 
Integrada (PPI), e possibilitaram a reorganização dos processos de gestão e de regulação do sistema de saúde no âmbito dos estados, com vistas à melhoria do acesso do cidadão à rede de assistência (BRASIL, 2011a).

Os municípios de uma microrregião, ao se associarem, constituem um sistema microrregional de serviços de saúde, sob a forma de uma rede de atenção à saúde (RAS), com base na cooperação gerenciada (MINAS GERAIS, 2004). "A RAS, definida como o conjunto de ações e serviços de saúde articulado em níveis de complexidade crescente, tem por finalidade garantir a integralidade da assistência à saúde" (BRASIL, 2015, p. 30).

A integralidade, segundo Sala et al. (2011), considera a rede de serviços composta por distintos níveis de complexidade e de competências, na qual a integração entre as ações satisfaz o conjunto de cuidados demandados pelo indivíduo.

De acordo com Kamimura e Oliveira (2013) a organização dos serviços de saúde deve obedecer a uma hierarquia, em forma de rede, de modo que as questões menos complexas sejam atendidas em local de menos recursos, partindo das unidades básicas de saúde, passando pelas unidades especializadas, pelo hospital geral, até chegar ao hospital especializado.

Assim, o modelo em redes assistenciais hierarquizadas busca garantir a integralidade da atenção à saúde dos usuários por meio do acesso a todos os níveis de complexidade, sendo que, esse acesso "inicia-se e se completa na Rede de Atenção à Saúde, mediante referenciamento do usuário na rede regional e interestadual, conforme pactuado nas Comissões Intergestores" (BRASIL, 2011c, p. 2).

A programação da atenção básica é de responsabilidade exclusiva dos municípios não estando previstos referenciamentos. Já para a média complexidade ambulatorial, a lógica de programação é ascendente, sendo que os municípios programam as ações de sua população e realizam os encaminhamentos para outros municípios, daquelas ações que não possuem oferta, por insuficiência ou inexistência de capacidade instalada (BRASIL, 2006b).

Mato Grosso do Sul, acompanhando os ditames nacionais, vem participando ativamente na implementação do SUS ao longo dos anos (DOBASHI, 2014). A princípio cumprindo as Normas Operacionais Básicas (NOB), seguidas pelas Normas Operacionais de Assistência à Saúde (NOAS), passando pelo Pacto pela Saúde até chegar ao Contrato Organizativo de Ação Pública de Saúde (COAP).

Salienta-se que Mato Grosso do Sul foi um dos primeiros estados a celebrar o Pacto pela Saúde com a adesão de 100\% dos 78 municípios existentes à época (SMAKA, 2013) e, também, o primeiro estado a assinar o COAP com 100\% de seus municípios (LEWANDOWSKI; BARROS, 2014).

Apesar da importância da PPI na organização e gestão do SUS, o conhecimento acerca do papel que esse instrumento de planejamento teria na garantia da integralidade da atenção à saúde no estado de Mato Grosso do Sul, é incipiente.

Buscando avançar nessa direção, a pesquisa teve como objetivo analisar a PPI como instrumento de garantia da integralidade da atenção à saúde da população em oito municípios pertencentes às quatro regiões de saúde do estado de Mato Grosso do Sul, na visão dos atores sociais envolvidos na sua elaboração e execução. Nessa perspectiva, buscou-se identificar os fatores que influenciam a execução da PPI no âmbito intermunicipal e sua repercussão sobre o processo de compartilhamento da gestão do SUS, partindo-se do pressuposto de que a pactuação e contratualização dos serviços garante o acesso da população a todos os níveis de atenção. 


\section{METODOLOGIA}

Foi realizada uma pesquisa qualitativa exploratória realizada mediante entrevistas semiestruturadas visando identificar se a PPI se constituía em um instrumento de garantia do acesso da população a todos os níveis de atenção.

O universo de análise foi composto pelos municípios de Mato Grosso do Sul distribuídos nas quatro Regiões de Saúde, e a amostra, escolhida por conveniência, foi composta pelos municípios que apresentaram diferenças na pactuação dos parâmetros de: cobertura da população alvo; concentração de procedimentos; e, cobertura da assistência, relativas à área estratégica "Saúde do Adulto - Hipertensão" em relação aos parâmetros estabelecidos pela Comissão Intergestores Bipartite (CIB) para o Estado.

Para fazer parte da amostra, os municípios preencheram os seguintes requisitos: ter pactuado três parâmetros diferentes dos propostos pela CIB para o estado de Mato Grosso do Sul (porcentual de cobertura da população alvo; concentração de procedimentos; e cobertura da assistência); cobrir as quatro Regiões de Saúde do Estado; e apresentar portes populacionais diferentes, desde que existentes na região.

Dessa forma, dos 78 municípios que compõem as quatro Regiões de Saúde, foram selecionados oito municípios, sendo três da Região de Saúde de Campo Grande; um da Região de Saúde de Corumbá; três da Região de Saúde de Dourados; e um da Região de Saúde de Três Lagoas.

Foram selecionados os municípios que alteraram os parâmetros de programação, considerando que aqueles que o fizeram tinham como objetivo adequá-los à realidade local, de forma a poder oferecer os serviços necessários para garantir a integralidade da atenção à saúde, seja por meio de serviços próprios, seja encaminhando os casos para unidades de referência de maior complexidade quando não houvesse capacidade para atendimento pelo próprio município. Nesse sentido, o respeito ao princípio da integralidade da atenção à saúde preconizada pelo SUS passa pela garantia de atendimento aos pacientes referenciados para unidades de maior complexidade das redes de atenção à saúde existentes no SUS.

As informações relativas aos fatores que influenciaram a garantia de acesso da população aos diversos níveis da atenção à saúde resultaram da aplicação de questionário semiestruturado, por meio de entrevista com roteiro previamente definido, mediante assinatura de Termo de Consentimento Livre e Esclarecido (TCLE).

As questões diziam respeito ao processo de planejamento/programação, identificação de fatores que influenciaram a pactuação, garantia de acesso, monitoramento e avaliação. 0 roteiro foi composto por perguntas fechadas e abertas que permitiram qualificar o processo de construção da programação e do referenciamento regional.

Constituíram-se em sujeitos da pesquisa os Secretários Municipais de Saúde (gestores) e técnicos das Secretarias Municipais de Saúde (SMS) que participaram do processo de elaboração da Programação Pactuada e Integrada da Assistência à Saúde que constou como anexo ao COAP 2012-2015 e suas atualizações.

O instrumento de pesquisa foi preenchido em entrevistas realizadas nos municípios da amostra, no período de 10 a 30 de julho de 2016. As entrevistas foram gravadas e, posteriormente, transcritas e analisadas. Procedeu-se à descrição das falas, com destaque para as similaridades e diferenças entre os relatos.

Para a apresentação dos resultados, os informantes receberam os seguintes códigos: uma letra que identifica a posição do entrevistado no sistema de saúde, sendo "G" para gestor 
municipal, e "T" para técnico; e, as letras $A, B, C, D, E, F, G$ e H, representando cada um dos municípios estudados.

O projeto de pesquisa foi aprovado pelo Comitê de Ética da Universidade Federal de Mato Grosso do Sul, conforme Parecer n. 1.613.750, de 29 de junho de 2016.

\section{RESULTADOS E ANÁLISES}

Foi possível observar, nas entrevistas, que o processo de pactuação nas regiões de saúde do estado de Mato Grosso do Sul contou com a adesão de $100 \%$ de seus municípios e foi realizado de forma ascendente, respeitando-se o Plano Diretor de Regionalização/PDR do Estado, sendo que os gestores e/ou técnicos municipais participaram de todas as etapas do processo.

Acrescenta-se, ainda, que a Secretaria de Estado de Saúde (SES) realizou uma série de reuniões e oficinas que contaram com a participação de gestores, técnicos, trabalhadores da saúde e comunidade, com vistas a discutir e orientar sobre os instrumentos de planejamento e programação.

Cabe aos gestores definir as prioridades que orientam a programação das ações e serviços de saúde de seus municípios (BRASIL, 2015), com base na análise da situação de saúde, peculiaridades e necessidades locorregionais (BRASIL, 2006a). Entretanto observou-se, nas respostas apresentadas, que a identificação da demanda e a definição das ações e serviços de saúde pactuados não resultaram da realização de diagnósticos situacionais, mas da utilização da série histórica dos atendimentos. Verifica-se que os municípios cumprem as regras estabelecidas, mesmo que estas limitem a sua liberdade de planejamento de acordo com as necessidades locais e comprometam a integralidade da assistência.

Dessa forma, caso a primeira pactuação tenha sido estabelecida muito abaixo de sua realidade local, o município terá, como consequência da adoção desse procedimento, a perpetuação de eventuais déficits de atendimento.

A PPI é o instrumento por meio do qual são definidas e quantificadas as ações de saúde para a população residente em cada território, tendo a conformação de redes de serviços regionalizadas sido pensada como uma forma de garantir o acesso da população a todos os níveis de atenção (BRASIL, 2006b). Cabe ao gestor municipal garantir que a população sob sua responsabilidade tenha acesso à atenção básica e aos serviços especializados (de média e alta complexidade), mesmo quando localizados fora de seu território (BRASIL, 2009).

Pelas respostas apresentadas, identificou-se que os oito municípios ouvidos garantem o acesso de sua população no nível de atenção básica, cujo atendimento é de responsabilidade do município. No entanto, com relação às especialidades, que são referenciadas, ou seja, naquelas ações em que os munícipes são direcionados a outro município para atendimento, nem sempre o acesso está garantido.

Segundo a técnica do município " $A$ ", os serviços referenciados não estão sendo atendidos de acordo com o pactuado. Ela afirmou que os municípios para os quais os serviços são referenciados não mantêm um controle efetivo sobre o que já foi atendido e o que ainda falta atender: "Você sabe quanto foi pactuado, [...] mas não sabe quem pactuou o que e, quanto é de cada município" (TA).

O próprio município não controla o quanto já encaminhou do pactuado, não havendo acompanhamento por parte do município referenciador, tampouco do executor:

Não há um acompanhamento entre os municípios com relação ao planejamento e o que está sendo executado. Falta controle entre a PPl e o executado com relação à referência (TA).

INTERAÇÕES, Campo Grande, MS, v. 18, n. 4, p. 99-108, out./dez. 2017. 
No nível da atenção básica, o município atende mais do que o foi pactuado, mas, nos casos das especialidades, não:

O município pactua com você que vai receber teste ergométrico, por exemplo, mas ele não sabe nem a capacidade dele, nem dá conta da capacidade dele, como que ele vai te ofertar? A PPI não é hoje uma garantia de acesso. Você pactua, mas não tem o serviço garantido (TC).

O mesmo ocorreu no município " $\mathrm{D}$ ", onde, segundo a técnica, a pactuação das referências não é feita pela demanda do município referenciador, mas pela disponibilidade de cumprimento por parte do município executor. Ela afirmou que se chega "na pactuação e o município fala o que tem para oferecer, e nós fazemos os ajustes de acordo com o ofertado". Afirmou, ainda, que a PPI não garante o atendimento, pois o município executor declina da prestação dos serviços dizendo não ter vaga:

A gente leva a demanda e o município diz que não pode me atender, aí eu pergunto: quanto pode atender? Posso atender tanto. Assim, ajustam-se os valores e pactua-se dessa forma (TD).

A gestora do município "E" também afirmou garantir, para a atenção básica, 100\% ou mais dos serviços pactuados, não sendo garantidos, entretanto, os serviços referenciados. Ela disse que pactua o que o município necessita, mas não tem certeza de que será atendida:

Quando vai referenciar o outro tem que aceitar, tem que ser vista a capacidade de atendimento do município que vai receber, pois ele não é obrigado a aceitar. Às vezes aceitam minha referência depois não me atendem. Posso afirmar que nas especialidades o atendimento não chega nem a $50 \%$ do pactuado (GE).

A técnica do município " $F$ " também informou não ter garantia de atendimento dos serviços referenciados:

Pactuamos com Dourados e Campo Grande e pode ocorrer de não ter transporte para levar o paciente e, se o paciente não tiver condições de se deslocar, acabamos perdendo a vaga. Pode acontecer, também, de o município não atender (TF).

A técnica do município " $G$ " afirmou que a PPI não garante o acesso da população e que, em sua opinião, o que garante o acesso é a organização do processo de trabalho no município: "Acho que o principal não é o que está pactuado, é o processo de trabalho do município que tem que ser organizado na questão de planejamento" (TG).

Para a referida técnica, a PPI constitui-se em um norte, mas não garante que o que está pactuado vai acontecer: "A PPI é importante, pelo menos a gente tem um parâmetro, te norteia para você se programar e elaborar o seu processo de trabalho" (TG).

A entrevistada afirmou que os exames de especialidades não são cobertos pelo município e sim pela rede particular e, o que é referenciado, às vezes, não é atendido: "Se não atende, cadê o profissional que acompanha? Como eu sei o quanto me atendeu?" (TG).

A efetividade da PPI como mecanismo de pactuação e organização da assistência nos municípios e regiões, para garantir o acesso equânime da população aos diversos níveis de assistência, requereria também um processo sistemático de acompanhamento da execução da programação e aperfeiçoamento dos próprios instrumentos e processos de negociação (MOLESINI et al., 2010). Entretanto foi possível perceber, nas entrevistas, que os municípios não acompanham a execução da PPI, não sabendo, portanto, o que está acontecendo, o que já foi feito e, simplesmente, não tem como fazer cobranças. 
De acordo com o técnico do município "H", têm-se os dois lados: para o município que faz a pactuação, é mais formal, pois ele quer a consulta e/ou o exame, entretanto não garante para o paciente, pois ele não tem a certeza de que será atendido. O entrevistado afirmou que "para garantia teria que pegar a PPI, tabular e contratar, ver a rede".

Como nos demais municípios, o técnico do município " $H$ " alegou que "o atendimento em atenção básica é garantido, por ser prestado no próprio município, sendo feito até mais que o programado, mas nas especialidades não é garantia, pois não sei se o município vai ter esse serviço". O técnico afirmou que: "é feita a pactuação, vai para o SISREG (Sistema de Regulação) e quando peço o serviço o município executor vai ver se ele tem a vaga, se não tiver ele não me atende, outras vezes diz não ter o profissional, e aí não me atende".

O técnico do município " $\mathrm{H}$ " falou com propriedade sobre a falta de garantia de acesso nos serviços referenciados, pois o seu município é referência em Psiquiatria para outros municípios:

Está pactuado entre nós e eles estão mandando, mas o profissional pediu as contas e eu não consigo atender, pois não consegui contratar outro profissional. Pactuei porque na época eu tinha o profissional. Não é uma garantia, hoje não é, deveria ser, mas não é (TH).

O município "B" não referencia serviços, todavia atende referências de todos os municípios do Estado. Fica, assim, responsável pela regulação das vagas próprias e das que the são referenciadas.

Segundo o técnico de "B" a PPI, no caso das referências, não é garantia de acesso visto que nem tudo o que está pactuado será atendido: "A oferta é dinâmica, hoje tenho, amanhã posso não ter" (TB). Ele afirmou que, no caso da atenção básica, há garantia de atendimento, mas na urgência, emergência e especialidades não consegue garantir o acesso por problema de estrutura.

O referido técnico afirmou, ainda, que na regulação das vagas o profissional regulador não leva em consideração o financeiro nem a cota que o município referenciador tem. O profissional analisa a solicitação que consta no sistema com base em protocolos clínicos, protocolos de regulação, fluxos pré-definidos e grades de referência, além da oferta disponível:

Se o município pactuou 100, ele pensa que tenho que garantir 100\% do que ele pactuou, independente de ele precisar ou não dessas vagas, mas para ser atendido o paciente que está sendo recebido deve preencher os critérios estabelecidos e analisados pelo profissional regulador (TB).

Tem município pequeno que pactua 10 e necessita de 20 ou 30. Se o município preenche os critérios, atendo os 20 ou 30 deles e só 20 do outro (TB).

O técnico de "B" relatou que não "trabalha com a oferta e não cuida se o município que pactuou encaminhou paciente no mês", destacando que o setor de regulação não cria, e sim gerencia o fluxo das ofertas para que todos tenham acesso aos atendimentos necessários.

A PPI é parte integrante do COAP, instrumento jurídico que explicita os compromissos pactuados em cada Região de Saúde (BRASIL, 2015) que, firmados pelos gestores, devem ser cumpridos. Entretanto planejar consiste em decidir algo com antecedência, de modo a alcançar objetivos propostos (KUSCHNIR; CHORNY; LIRA, 2010).

Sendo considerada uma previsão, o planejado pode ou não acontecer da forma programada, havendo a possibilidade de os acordos estabelecidos e contratualizados não serem atendidos, uma vez que o acesso às ações e aos serviços de saúde será fundado na avaliação da gravidade do risco individual e coletivo (BRASIL, 2011c).

Está errado? Há que se punir o município que contratualizou e aceitou referenciamentos e não os cumpriu? Se levar-se em consideração o documento formal assinado, que como princípio 
funciona como um instrumento legal que obriga as partes ao seu cumprimento, sim, os entes signatários devem cumprir estabelecido no contrato assinado e garantir a prestação da atenção aos pacientes do município referenciador.

Por outro lado, como deixar de atender um paciente que necessita de cuidados mais urgentes podendo, inclusive, estar correndo risco a vida dele, em detrimento de um acordo que apresente pacientes que podem esperar pelo atendimento?

Os resultados da pesquisa mostraram que, embora a PPI seja uma importante ferramenta de planejamento e de negociação entre os municípios, ela não está se constituindo em um instrumento de garantia de acesso da população a todos os níveis de atenção.

Os resultados mostraram, ainda, que o recurso financeiro é um fator limitador da prestação de serviços à população, uma vez que os recursos destinados ao pagamento das ações de atenção à saúde prestada entre municípios são alocados previamente pelo gestor que demanda esses serviços, ao município sede do executor (BRASIL, 1996), ou seja, o município executor incorpora os recursos relativos à prestação desses serviços ao seu teto financeiro.

Assim, o município que referencia os serviços paga pelos atendimentos referenciados antes da prestação deles pelo município executor, mesmo não tendo a garantia de que o paciente encaminhado será atendido. No caso de o serviço não ser prestado, o recurso se perde, não retornando ao município que o referenciou.

A PPI é, também, um instrumento por meio do qual são definidos os limites financeiros destinados à assistência da população própria e referenciada (BRASIL, 2006b). O limite financeiro da assistência por município é o montante máximo de recursos federais que poderá ser gasto com o conjunto de serviços existentes em cada território municipal (BRASIL, 2001). Constatouse, nesta pesquisa, que os limites financeiros, apesar da previsão de reprogramação, não vêm sendo atualizados, acarretando a manutenção dos quantitativos pactuados e o não atendimento da população referenciada.

\section{CONSIDERAÇÕES FINAIS}

O estado, por meio do oferecimento de treinamentos e capacitações diversas, deu condições e apoio necessários para os municípios implementarem a PPI. Apesar disso, constatou-se que o planejamento não é feito com base em diagnósticos atualizados da situação de saúde, mas na reprodução do passado, tendo como consequência o afastamento da realidade e a reprodução dos problemas/deficiências anteriores.

Verificou-se que a atenção básica é a porta de entrada da atenção à saúde, por meio da qual a população tem acesso universal e atenção integral. Isso é observado pelas afirmações dos entrevistados, que assumem que os municípios garantem o acesso às ações e serviços de saúde em atenção básica, executando, inclusive, quantitativos maiores do que os pactuados na PPI.

Já com relação à integralidade da atenção, não se verificou essa garantia. Os entrevistados afirmaram que, mesmo estando negociado e contratualizado, não há garantia de que a unidade de maior complexidade referenciada irá atender os casos encaminhados pelo município.

Convém frisar que a PPI se tem constituído em um instrumento essencialmente de pactuação de referências, necessário para garantir o aporte de recursos do SUS e não para um efetivo planejamento em saúde e garantia da integralidade da atenção. Para os municípios, pactuação é sinônimo de negociação entre os gestores para o estabelecimento de referências. 
Não há um controle por parte dos municípios de forma a identificar o que, dentre as ação e serviços pactuados, está efetivamente sendo realizado, razão pela qual, mesmo com procedimentos pactuados, o município pode não ser atendido.

Apesar de a PPI ser um processo pactuado e contratualizado, verificou-se que não há uma garantia de atendimento dos usuários pelas unidades executoras por razões como falta de capacidade instalada, falta de planejamento realista, situações não previstas, e pela existência de um conflito entre o respeito ao que foi legalmente acordado e o respeito à vida humana, expresso pela gravidade clínica dos casos recebidos na unidade de referência. O respeito à vida humana é o princípio que tem prevalecido no campo da saúde.

Verifica-se, assim, que a PPI não está cumprindo o seu papel de garantidora do acesso aos vários níveis de assistência, uma vez que não há garantia de que os serviços referenciados serão executados e, consequentemente, da integralidade da atenção, ocasionando a não resolutividade da demanda de saúde existente.

\section{REFERÊNCIAS}

AMORIM, André L. G.; MENICUCCI, Telma M. G. Descentralização e regionalização na assistência à saúde: um estudo da programação pactuada integrada de Minas Gerais. In: ENCONTRO DE ADMINISTRAÇÃO PÚBLICA E GOVERNANÇA (EnAPG), 3., 12-14 nov. 2008, Salvador, BA. Anais... Salvador: EnAPG. Disponível em: <http://www.anpad.org.br/diversos/trabalhos/EnAPG/enapg_2008/2008_ENAPG255.pdf>. Acesso em: 4 jan. 2016.

BRASIL. Conselho Nacional de Secretários de Saúde. A Gestão do SUS. Brasília: CONASS, 2015. 133p. Disponível em: <http://www.conass.org.br/biblioteca/pdf/A-GESTAO-DO-SUS.pdf>. Acesso em: 20 abr. 2016.

. Conselho Nacional de Secretários de Saúde. Sistema Único de Saúde. Brasília: CONASS, 2011a. 291p. (Coleção Para entender a gestão do SUS 2011, v. 1).

. Conselho Nacional de Secretários de Saúde. Legislação Estruturante do SUS. Brasília: CONASS, 2011b. 534p. (Coleção Para entender a gestão do SUS 2011, v. 13).

. Presidência da República. Decreto n. 7.508, de 28 de junho de 2011c. Regulamenta a Lei n. 8.080, de 19 de setembro de 1990, para dispor sobre a organização do Sistema Único de Saúde - SUS, o planejamento da saúde, a assistência à saúde e a articulação interfederativa e dá outras providências. Diário Oficial [da] República Federativa do Brasil, Brasília, DF, de 29 jun. 2011. Seção I, p. 1-3.

. Ministério da Saúde. Secretaria-Executiva. Departamento de Apoio à Descentralização. O SUS no seu município: garantindo saúde para todos. 2. ed., Brasília, DF: Ministério da Saúde, 2009. 46 p. (Série B. Textos Básicos de Saúde). Disponível em: <http://bvsms.saude.gov.br/bvs/publicacoes/sus_municipio_ garantindo_saude.pdf $>$. Acesso em: 20 jan. 2016.

. Ministério da Saúde. Secretaria Executiva. Subsecretaria de Planejamento e Orçamento. Sistema de planejamento do SUS: uma construção coletiva: perfil da atividade do planejamento no Sistema Único de Saúde: resultados da pesquisa - esfera municipal. Brasília: Ministério da Saúde, 2008. 142p. (Série B. Textos Básicos de Saúde; Série Cadernos de Planejamento, v. 5).

. Ministério da Saúde. Portaria GM/MS n. 399, de 22 de fevereiro de 2006a. Divulga o Pacto pela Saúde 2006 - Consolidação do SUS e aprova as Diretrizes Operacionais do referido Pacto. Diário Oficial [da] República Federativa do Brasil, Brasília, DF, 23 fev. 2006. Seção I, p. 43-51.

. Ministério da Saúde. Secretaria de Atenção à Saúde. Departamento de Regulação, Avaliação e Controle de Sistemas. Diretrizes para a programação pactuada e integrada da assistência à saúde. Brasília: Ministério da Saúde, 2006b. 148p. (Série B. Textos Básicos de Saúde; Série Pactos pela saúde, v. 5).

. Ministério da Saúde. Portaria GM/MS n. 95, de 26 de janeiro de 2001. Aprova Norma Operacional da Assistência à Saúde: NOAS-SUS 01/01. Diário Oficial [da] República Federativa do Brasil, Brasília, DF, 29 jan. 2001. Seção I, p. 23-31.

INTERAÇÕES, Campo Grande, MS, v. 18, n. 4, p. 99-108, out./dez. 2017. 
. Ministério da Saúde. Portaria GM/MS n. 2.203, de 5 de novembro de 1996. Aprova a Norma Operacional Básica do SUS- NOB 01/96. Diário Oficial [da] República Federativa do Brasil, Brasília, DF, 6 nov. 1996. Seção I, p. 22932-40.

Presidência da República. Lei n. 8.080, de 19 de setembro de 1990. Dispõe sobre as condições para a promoção, proteção e recuperação da Saúde, a organização e o funcionamento dos serviços correspondentes e dá outras providências. Disponível em: <http://www.planalto.gov.br/ccivil_03/leis/ L8080.htm>. Acesso em: 15 mar. 2015.

Presidência da República. Constituição da República Federativa do Brasil, de 5 de outubro de 1988. Disponível em: <www.planalto.gov.br/ccivil_03/constituicao/constituicao.htm>. Acesso em: 15 mar. 2015.

DOBASHI, Beatriz F. (Org.). Tecendo redes na saúde para ampliar o cuidado. Apresentação. Campo Grande, MS: [s.n.], 2014. p. 5-9.

KAMIMURA, Quésia P.; OLIVEIRA, Edson A. A. Q. Processo de regionalização da saúde na percepção dos gestores. Revista Brasileira de Gestão e Desenvolvimento Regional, v. 9, n. 2, p. 280-300, maio/ ago. 2013, Taubaté, SP, Brasil. Disponível em: <http://www.rbgdr.net/revista/index.php/rbgdr/article/ viewFile/1030/339>. Acesso em: 27 out. 2014.

KUSCHNIR, Rosana C.; CHORNY, Adolfo H.; LIRA, Anilska M. L. Gestão dos sistemas e serviços de saúde. Florianópolis: Departamento de Ciências da Administração/UFSC; Brasília: CAPES; UAB, 2010. 180p.

LEWANDOWSKI, Bernardete G.; BARROS, Eugênio O. M. História do SUS em Mato Grosso do Sul. In: DOBASHI, Beatriz F. (Org.). Tecendo redes na saúde para ampliar o cuidado. Campo Grande, MS, 2014. p. 28-44.

MINAS GERAIS, Estado [de]. Secretaria de Estado de Saúde. Pacto de gestão: da municipalização autárquica à regionalização cooperativa. Marcus Vinícius Caetano Pestana da Silva e Eugênio Vilaça Mendes (Org.). Belo Horizonte: Secretaria de Estado de Saúde, 2004. 80p.

MOLESINI, Joana A.; FORMIGLI, Vera L. A.; GUIMARÃES, Maria C. L.; MELO, Cristina M. M. Programação pactuada integrada e gestão compartilhada do SUS. Revista Baiana Saúde Pública, v. 34, n. 3, p. 623-38, jul./set. 2010.

SALA, Arnaldo; LUPPI, Carla G.; SIMÕES, Oziris; MARSIGLIA, Regina G. Integralidade e atenção primária à saúde: avaliação na perspectiva dos usuários de unidades de saúde do município de São Paulo. Saúde e Sociedade, São Paulo, v. 20, n. 4, p. 948-960, 2011.

SILVA, Silvio F. da. Organização de redes regionalizadas e integradas de atenção à saúde: desafios do Sistema Único de Saúde (Brasil). Ciência \& Saúde Coletiva, Rio de Janeiro, v. 16, n. 6, p. 2753-62, 2011.

SMAKA, Emir M. Case de sucesso: Secretaria de Estado de Saúde de Mato Grosso do Sul desenvolve solução para a tomada de decisões estratégicas. Portal IT4CIO, Curitiba, maio de 2013. Disponível em: <http://www.it4cio.com/resources/images/materias/pdfs/471.pdf>. Acesso em: 20 jan. 2016.

VENANCIO, Sonia I.; NASCIMENTO, Paulo R. do; ROSA, Tereza E.; MORAIS, Maria de L. S. e; MARTINS, Patrícia N.; VOLOSCHKO, Anna. Referenciamento regional em saúde: estudo comparado de cinco casos no estado de São Paulo, Brasil. Ciência \& Saúde Coletiva, Rio de Janeiro, v. 16, n. 9, p. 3951-64, 2011.

\section{Sobre os autores:}

Lenice Carrilho de Oliveira Moreira: Administradora. Mestre em Tecnologias Ambientais pela Universidade Federal de Mato Grosso do Sul (UFMS). Doutoranda em Saúde e Desenvolvimento na Região Centro-Oeste da Universidade Federal de Mato Grosso do Sul. E-mail: lenice.moreira@ufms.br

Edson Mamoru Tamaki: Administrador (EAESP/GV). Doutorado em Economia da Saúde (Universidade de Paris IX) e Pós-doutorado em Saúde Pública (Faculdade de Saúde Pública/USP). Professor Titular do Centro de Ciências Biológicas e da Saúde da UFMS. Área de atuação: Saúde Coletiva. Área de Pesquisa: Avaliação e Gestão em Saúde. E-mail: edson.tamaki@ufms.br 